\title{
Magnetotail response during a strong substorm as observed by GEOTAIL in the distant tail
}

\author{
A. Belehaki ${ }^{1}$, R. W. McEntire ${ }^{2}$, S. Kokubun ${ }^{3}$, T. Yamamoto $^{4}$ \\ ${ }^{1}$ Institute of Space Research, National Observatory of Athens, Metaxa and Vas. Pavlou Str., Palaia Pendeli, GR-152 36, Greece \\ 2 The Johns Hopkins University, Applied Physics Laboratory, Laurel, MD 20723, USA \\ ${ }^{3}$ Solar Terrestrial Environment Laboratory, Nagoya University, Toyokawa Aichi, 442, Japan \\ ${ }^{4}$ Institute of Space and Astronautical Science, Sagamihara, Kanagawa 229, Japan
}

Received: 24 July 1997 / Revised: 5 December 1997 / Accepted: 8 December 1997

\begin{abstract}
Simultaneous energetic particle and magnetic field observations from the GEOTAIL spacecraft in the distant tail $\left(X_{G S M} \approx-150 \mathrm{Re}\right)$ have been analysed to study the response of the Earth's magnetotail during a strong substorm $(\mathrm{AE} \leq 680 \mathrm{nT})$. At geosynchronous altitude, LANL spacecraft recorded three electron injections between 0030 UT and 0130 UT, which correspond to onsets observed on the ground at Kiruna Ground Observatory. The Earth's magnetotail responded to this substorm with the ejection of five plasmoids, whose size decreases from one plasmoid to the next. Since the type of magnetic structure detected by a spacecraft residing the lobes, depends on the $Z$ extent of the structure passing underneath the spacecraft, GEOTAIL is first engulfed by a plasmoid structure; six minutes later it detects a boundary layer plasmoid (BLP) and finally at the recovery phase of the substorm GEOTAIL observes three travelling compression regions (TCRs). The time-of-flight (TOF) speed of these magnetic structures was estimated to range between $510 \mathrm{~km} / \mathrm{s}$ and $620 \mathrm{~km} / \mathrm{s}$. The length of these individual plasmoids was calculated to be between $28 \mathrm{Re}$ and $56 \mathrm{Re}$. The principal axis analysis performed on the magnetic field during the TCR encountered, has confirmed that GEOTAIL observed a 2-D perturbation in the $X-Z$ plane due to the passage of a plasmoid underneath. The first large plasmoid that engulfed GEOTAIL was much more complicated in nature probably due to the external, variable draped field lines associated with high beta plasma sheet and the PSBL flux tubes surrounding the plasmoid. From the analysis of the energetic particle angular distribution, evidence was found that ions were accelerated from the distant $X$-line at the onset of the burst associated with the first magnetic structure.
\end{abstract}

Key words. Magnetospheric physics (magnetospheric configuration and dynamics; magnetotail).

\section{Introduction}

The magnetospheric response during substorm disturbances has been studied extensively over the last two decades using ground-based, geosynchronous satellite and spacecraft data sets. Several models have been proposed to describe the changes taking place in the magnetotail during substorms. Current magnetospheric models predict the formation of a plasmoid (Hones, 1976, 1979) in the near-Earth plasma sheet at the time of substorm onset. A plasmoid is a three-dimensional segment of the plasma sheet which is ejected down the magnetotail following substorm onset. According to Hones' (1976) model, the plasmoid consists of loops of closed magnetic field lines formed by pinching off plasma sheet magnetic field lines at a reconnection line. Once lobe magnetic field lines begin to merge at the same line, the plasmoid is ejected tailward. The conventional plasmoid is depicted in the $X-Z$ meridional plane and is, therefore, two dimensional. Hughes and Sibeck (1987) proposed that if a dawn to dusk magnetic field component is included in the standard plasmoid model, the resulting magnetic structure is a flux rope. In the 3D model, reconnected plasma sheet field lines form a magnetic flux rope-like structure, which stretches across the tail, but whose ends are connected to the auroral ionosphere. When open lobe field lines reconnect at the near-Earth neutral line, they form loops of flux that are topologically linked around the flux rope and which will tend to pull the flux rope tailward. Nevertheless the flux rope remains attached to the Earth until further reconnection near the flanks of the tail releases them. According to Hughes and Sibeck (1987) the particle signatures expected in such structures are similar to those observed in closed loop plasmoids. 
There have been a number of supporting observations such as high speed tailward flows in the plasma sheet, bipolar signature in the $B z$ and/or $B y$ component, and energetic ion anisotropies, to verify the consistency of these models. These signatures have been reported from the analysis of ISEE3 and GEOTAIL observations and verify the envelopment of the spacecraft by a bulge in the plasma sheet (Hones et al., 1984a,b; Scholer et al., 1984a,b,c; Zwickl et al., 1984; Richardson and Cowley, 1985; Baker et al., 1987; Richardson et al., 1987; Slavin et al., 1989; Moldwin and Hughes, 1992a; Machida et al., 1994; Frank et al., 1994). Moreover GEOTAIL observations supporting the existence of flux ropes include unidirectional electron distributions (Belehaki et al., 1996; 1997) and modification of the compositional content of energetic ions inside the structure (Lui et al., 1994).

A very important conclusion revealed from these studies is that the magnetic field and plasma variations detected by a spacecraft due to a passing plasmoid depend on the spacecraft trajectory relative to the plasmoid. If the spacecraft is engulfed by a passing plasmoid, the magnetometer signature will include a bipolar tilting in the $B z$ and/or $B y$ component and a distinct core as the spacecraft samples the core of the structure. If the spacecraft is located in the lobe in such a position so it does not penetrate the plasmoid, it will observe a travelling compression region. Travelling compression regions (TCRs) are several minute-long enhancements of the magnetic field intensity in the lobes of the tail, which are accompanied by a north-thensouth variation in the $B z$ component (Slavin et al., 1984). According to Slavin et al. (1989), a TCR is detected when a spacecraft follows a trajectory, such as in Fig. 1, which is too high in the $Z$ coordinate to encounter the plasmoid proper. As shown in the lower panels of Fig. 1, the lobes are very low beta regions, and the pulse of increase $|\mathrm{B}|$ peaks at the inflection point in a north - then - south tilting in the field. For a trajectory passing closer to the midplane of the plasmoid, i.e., $\mathrm{C}$ in Fig. 1, the situation is expected to be somewhat different. With the spacecraft remaining in relatively high beta plasma throughout the encounter, it might be expected that thermal pressure would play a dominant role in supporting the enhanced solar wind pressure and only a weak enhancement in $|\mathrm{B}|$ would be observed in association with the bipolar $B z$ signature of the plasmoid. For trajectories between $\mathrm{A}$ and $\mathrm{C}$ a significant peak in $|\mathrm{B}|$ might be observed.

During a survey of magnetometer and electron plasma data obtained during the ISEE3 Geotail Mission for plasmoid signatures in the plasma sheet (Moldwin and Hughes, 1992b) bipolar signatures were reported in the plasma sheet boundary layer (PSBL). The PSBL is primarily characterised by low-energy field aligned streaming ions. Secondary characteristics such as ion cyclotron waves produced by the ion beams and a decrease in magnetic field strength due to diamagnetic effect of the PSBL plasma could be observed (Tsurutani et al., 1985). The bipolar signatures reported in this magnetospheric region, are the TCR analog in the
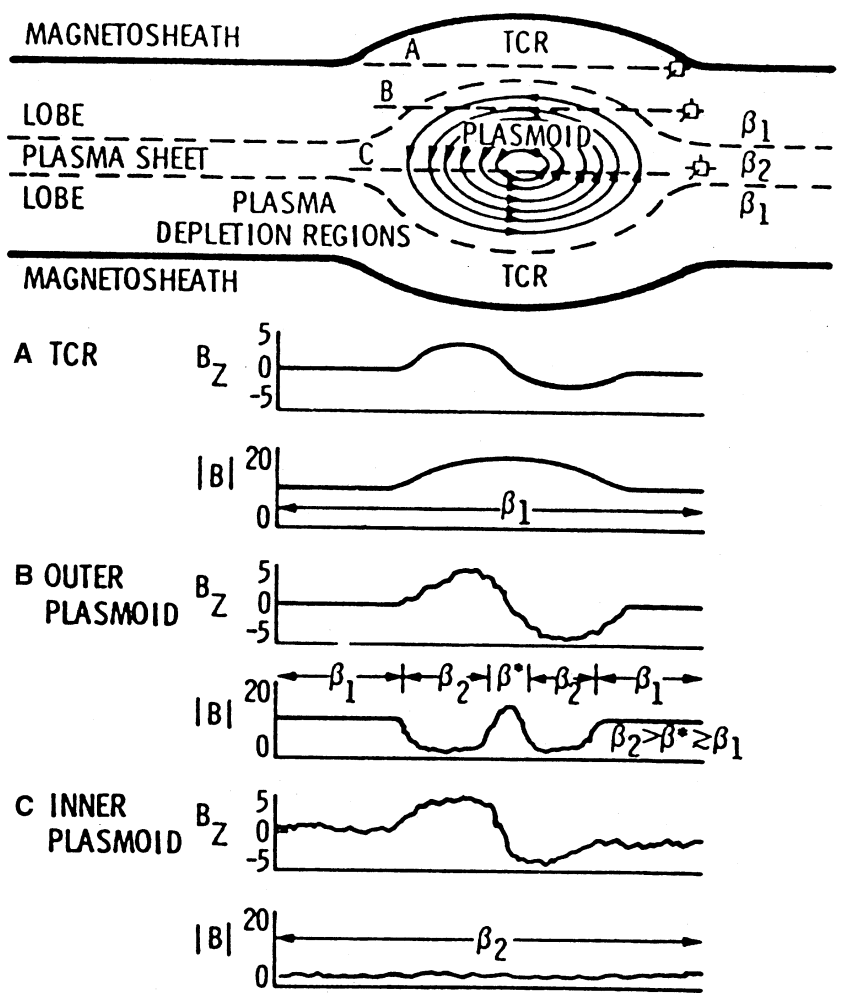

Fig. 1. Magnetic field and plasma beta variations observed along several different trajectories relative to an idealised plasmoid (from Slavin et al. 1993)

PSBL, that is they are due to the passage of a plasmoid. PSBL "plasmoids", known as boundary layer plasmoids (BLP), are rare. A BLP signature is expected only when the size of a plasmoid and the position of the spacecraft causes the spacecraft to just enter the thin PSBL (the PSBL is estimated to be only a few Re thick, Owen and Cowley, 1987) and not directly into the plasma sheet and the plasmoid. Moldwin and Hughes (1992b) have defined precisely the characteristics of a BLP: (1) bipolar signature in the $B z$ or $B y$ component and accompanying variations in the other two components; (2) decrease of magnetic field strength; (3) the electron temperature increases to just above $10^{6} \mathrm{~K}$ during the event; (4) the electron flow velocity rises from $\sim 200 \mathrm{~km} / \mathrm{s}$ to $\sim 800 \mathrm{~km} / \mathrm{s}$; and (5) clear substorm onset in ground magnetograms. Moreover, in two of the three BLP presented in detail by Moldwin and Hughes (1992b) some very small amplitude ion cyclotron waves were observed prior to and after the event. The primary characteristic of the PSBL, which is the low-energy field aligned streaming ions, could not be observed since ISEE3 had no ion plasma detector in operation.

An alternative picture to the classic plasmoid model of Hones (1976) concerning the magnetotail response after the onset of the magnetospheric substorm, was proposed by Slavin et al. (1993). In a comprehensive survey of TCR properties determined by the ISEE3 magnetotail measurements they suggested that the Earth's magnetotail response, after the onset of a substorm, depends on the intensity of the substorm 
determined by the AL index. They found that the intensity of the AL index corresponding to isolated TCR events was seen to be significantly lower than during the multiple TCR events. Hence while the magnetosphere dissipates larger quantities of energy during very intense substorms, it does so by ejecting multiple, smaller plasmoids rather than through the formation and expulsion of a single large plasmoid.

Some interesting studies of isolated events detected by ISEE3 and GEOTAIL during specific trajectories helped us to clarify the signatures of different types of structures. Among them, Murphy et al. (1987) studied the particle signatures of high energy ions detected by ISEE3 in the distant plasma sheet boundary layer. These ion enhancements were associated with magnetic field signatures of a travelling compression region. They suggested that the bulk movement of the plasma sheet, relative to the spacecraft, caused by the tailward motion of a plasmoid, moves the energetic ion boundary layer past the spacecraft. They showed that the $90^{\circ}$ pitch angle data (dawnward and duskward directed ions) support this idea fully, and so they concluded that the TCR ion bursts are caused by motion of an energetic ion boundary layer past the spacecraft.

Kawano et al. (1994) studied a flux rope detected by GEOTAIL in the middle tail, followed by bipolar $B z$ events identifiable as travelling compression regions. During the TCRs, the $\mathrm{E} \times \mathrm{B}$ flow showed a bipolar north-then-south perturbation, consistent with expected lobe plasma motion. The TCRs corresponded to weak ground magnetic disturbances and $\mathrm{Pi} 2$ waves. In order to explain these features they proposed that the flux rope and TCRs correspond to quasi-stagnant plasmoids, between the mid-tail and the distant $X$-lines, which are recurrently generated and moved slowly down the tail.

The aim of this study is to determine the dynamics of the magnetotail during an intense substorm. For this purpose we have selected a period of enhanced geomagnetic activity, when GEOTAIL was in a suitable position in the distant tail $(|X| \approx 150 \mathrm{Re})$ to detect various types of magnetic structures ejected after the onset of the expansion phase. The event presented here, occurred on October 10, 1993 from 0050 UT to 0220 UT. The study of this case shows clearly that a series of plasmoids are generated in the tail, with each roughly corresponding to a substorm intensification, and each being smaller than the previous one. The comprehensive new observations presented here are in contradiction to the classic plasmoid model (Hones, 1976) and give full support to the model of multiple plasmoid ejection (Moldwin and Hughes, 1993; Slavin et al., 1993).

\section{GEOTAIL instrumentation}

Energetic particle measurements used in this study are from the energetic particles and ion composition (EPIC) experiment (Williams et al., 1994). The instrument performs three-dimensional distribution measurements by using both total energy (LEMS, low energy compo-
Table 1. EPIC/ICS channels

\begin{tabular}{lcl}
\hline Channel & Resolution & Description \\
\hline E3 & $6 \mathrm{~s}$ & All ions of $62-74$ \\
& & keV $\left(\right.$ Mostly $\left.\mathrm{H}^{+}\right)$ \\
E4 & $6 \mathrm{~s}$ & All ions of $74-89 \mathrm{keV}\left(\mathrm{Mostly} \mathrm{H}^{+}\right)$ \\
E5 & $48 \mathrm{~s}$ & All ions of $89-110 \mathrm{keV}\left(\mathrm{Mostly} \mathrm{H}^{+}\right)$ \\
ED1 & $3 \mathrm{~s}$ & Electrons $>38 \mathrm{keV}$ \\
\hline
\end{tabular}

sition system) and velocity/composition detectors (ICS, ion composition system), measuring ions and electrons with energies $>20 \mathrm{keV}$, and ions with energy $>8 \mathrm{keV} /$ nucleon, respectively. For the purposes of this work we used the following ICS channels: E3, E4, E5, and ED1. Table 1 presents an overview of the channel characteristics.

The magnetic field data were obtained by the fluxgate magnetometer (MGF) on board GEOTAIL at a rate of 16 vectors/s and are used with a resolution of $3 \mathrm{~s}$. The instrument is described by Kokubun et al. (1994).

\section{Observations}

On October 10, 1993 between 0050 UT and 0220 UT a sequence of magnetic structures were detected by GEOTAIL. At that time the spacecraft was located in the deep geotail with $X_{G S M}=-148.5 \mathrm{Re}, Y_{G S M}=$ $10.9 \mathrm{Re}$ and $Z_{G S M}=8.2 \mathrm{Re}$. Since the tail axis is aberrated by $4.5^{\circ}$ towards dusk, due to the Earth's orbital motion, the rotated $Y$ coordinate (i.e., $Y^{\prime}$ ) gives the most probable spacecraft location to be at $Y^{\prime}=0.4 \mathrm{Re}$. The time plot of the first part of this event is given in Fig. 2. We present differential energy fluxes of the energetic particle channels ED1, E5, E4 and E3, from the sunward sector (solid line) and the tailward sector (dotted line), together with magnetic field data of 3 s time resolution.

At the start of this interval GEOTAIL was located in the north lobe. From 0055 UT to 0115 UT GEOTAIL detected an energetic particle burst which is associated with significant magnetic field disturbances probably due to a large plasmoid. A small but well-defined bipolar signature is observed along the $Z$-component of the magnetic field having its inflection point at 0105:50 UT. The plasmoid core field has a strong $B y$ component at the inflection point of the bipolar signature. The large magnetic field perturbation, observed at the leading and the trailing field, is due to the pile up of the plasma sheet ahead of the plasmoid. An interesting feature of this event is that the magnitude of the magnetic field at the centre of the structure presents a peak at 0105:50 UT without exceeding or even reaching the adjacent lobe field.

Energetic particles show a burst-type increase. To study the flow of energetic particles compared to the magnetic field direction in more detail we present the angular distributions of energetic electrons and protons in Fig. 3a,b respectively. The ion intensity is plotted linearly versus viewing direction and normalised to the 


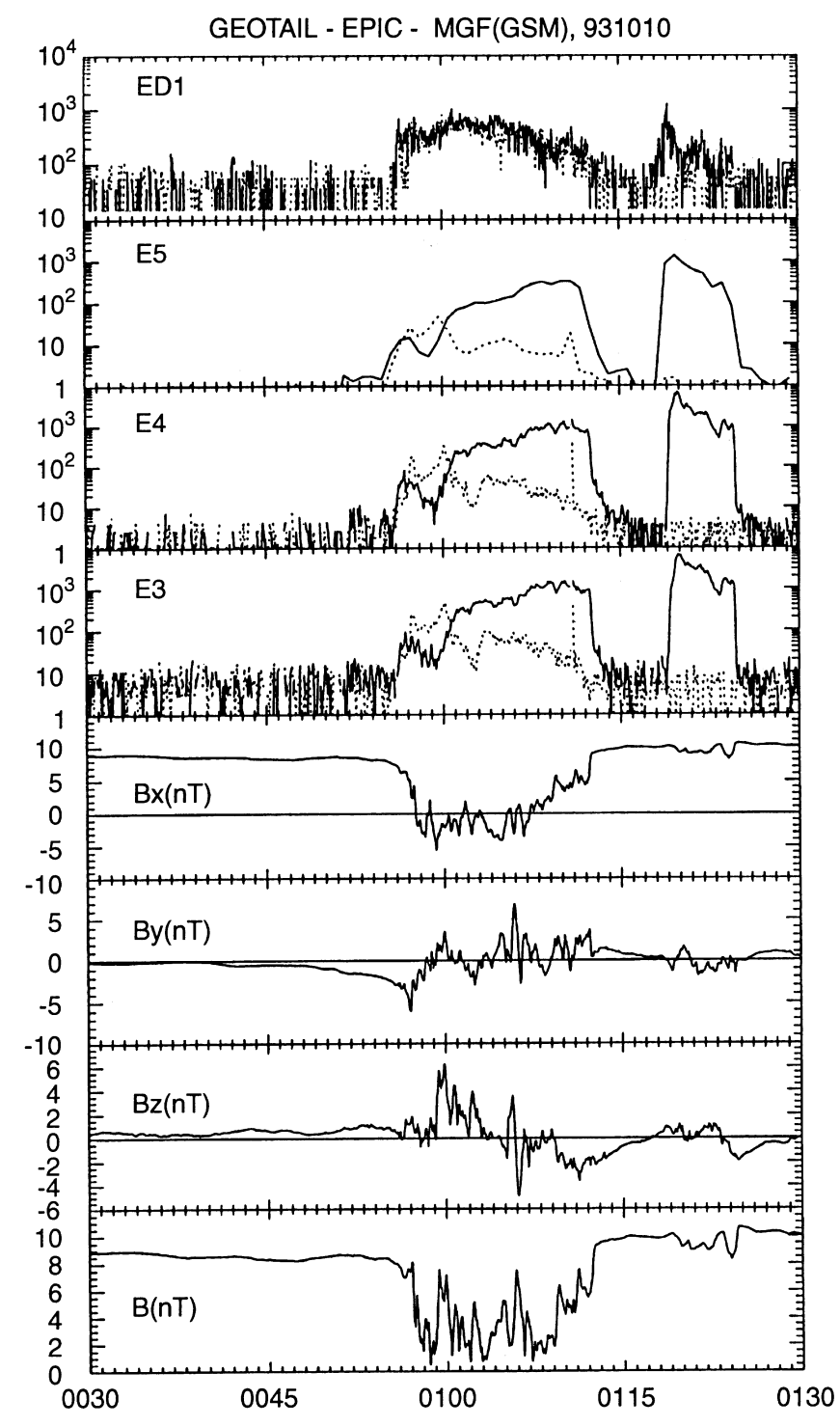

Fig. 2. The time plot of the magnetic field and energetic particle conditions for October 10, 1993 between 0030 UT and 0130 UT are presented. The top four panels show the differential energy fluxes of the energetic particle channels ED1, E5, E4, and E3. The bottom four panels show the magnetic field in GSM co-ordinates at 3 s resolution. For each channel the tailward (solid line) and earthward flux (dotted line) is presented

sector with maximum intensity. The Sun lies to the right of this figure. The time shown at the top of each plot is the start time of the $6 \mathrm{~s}$ accumulation interval. The number given at the top of each plot is the count rate (counts/s) of the sector with the maximum flux. Together with each angular distribution we overplot the equatorial vector of the mean value of the magnetic field during each $6 \mathrm{~s}$ time interval. The dashed line in each plot is the perpendicular to the field direction. The angular distributions of the energetic electrons with $\mathrm{E}>38 \mathrm{keV}$ are shown in Fig. 3a. GEOTAIL observes the first beam of energetic electrons at 0056:16 UT. Electrons are flowing tailward for almost $1 \mathrm{~min}$ and then their flow in the equatorial plane becomes isotropic. On the other hand, energetic ions present a different behaviour. The angular distributions of ions detected from channel E3 are presented in Fig. 3b. The first beam of ions is observed at 0057:16 UT to flow earthward along the field lines, indicating that the distant neutral line is formed tailward of GEOTAIL, that is at $|X|>148.5 \mathrm{Re}$. The earthward flow of E3 ions is observed until 0060:28 UT. Consequently, until 0101:16 UT a flow reversal to tailward direction is observed. In the tailward anisotropy a systematic crosstail component is observed, which lasts for $\sim 7 \mathrm{~min}$ providing evidence of a slow displacement of the PSBL across the spacecraft. The same pattern of flow is observed in the other two channels, E4 and E5, whose anisotropies have been examined in detail but not shown here. No evidence for energy dispersion was found in the front edge of this plasmoid structure.

To characterise the state of the magnetosphere prior to and during this event, we inspected the AE indices for October 9 and 10, 1993, which are presented in Fig. 4a. Two major onsets are identified at about 2100 UT and $2200 \mathrm{UT}$, on October 9, 1993, giving rise to a very strong substorm, which lasts until about 2330 UT of that day. At 0040 UT of October 10, 1993, the onset of a new substorm is detected from the AE indices, which we believe to be responsible for the large plasmoid observed by GEOTAIL from 0055 to 0112 UT. Since between the two substorms the AE index remains high enough $(\sim 300 \mathrm{nT})$, it is concluded that the substorm that caused the disturbance detected at the magnetotail is part of a longer period of disturbance.

To study in more detail the geomagnetic activity for October 10, 1993 between 0000 UT and 0230 UT, we present in the upper panel of Fig. $4 \mathrm{~b}$ the energetic electron fluxes from five different energy levels from LANL 1990-095 geosynchronous satellite, and the 1 min geomagnetic field disturbance data from Kiruna Observatory, in the lower panel. A notable onset is seen at 0036 UT at geosynchronous altitude, following by two intensifications at 0100 UT and 0116 UT (marked with arrows in Fig. 4b). Almost simultaneous decreases as seen in the Kiruna magnetogram.

One of the clearest markers of substorm onsets in high-altitude spacecraft data is the occurrence of these substorm particle injections near local midnight at geostationary orbit. Sharp dispersionless injection event onsets generally are associated one-to-one with substorm expansion onsets (or intensifications) seen in ground-based data (Baker et al., 1984). Thus, the substorm onset associated with the plasmoid detected by GEOTAIL at $0057 \mathrm{UT}$, was identified from the ground magnetogram of the Kiruna Observatory at $0040 \mathrm{UT}$, that is $\sim 17 \mathrm{~min}$ prior to magnetic structure appearance at GEOTAIL position.

The passage of the first plasmoid leaves GEOTAIL in a region of constant magnetic field whose value is $10 \mathrm{nT}$, i.e., higher than the north lobe field measured before the first plasmoid encountered, by $\sim 25 \%$. Under these conditions, a second plasmoid-like perturbation is encountered, $7 \mathrm{~min}$ after the passage of the first plasmoid. An expanded view of this second perturbation is presented in Fig. 5. The format is the same as the one 

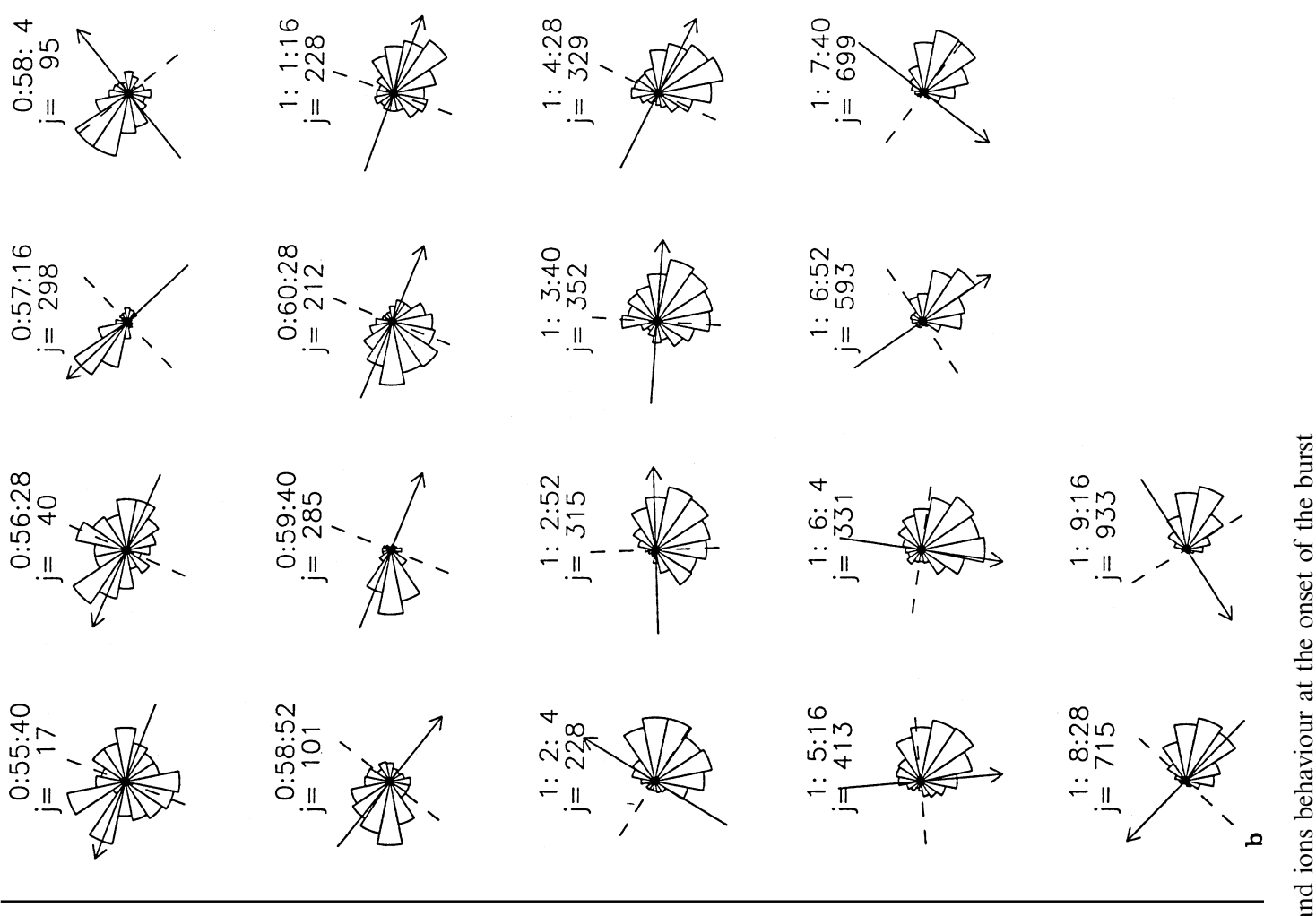

$\stackrel{\infty}{\sim} \sim$

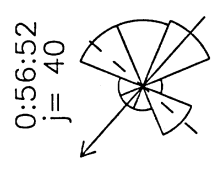

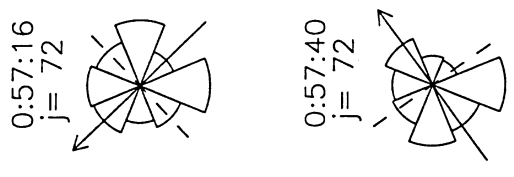

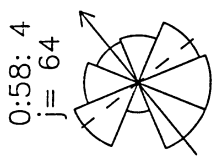

-
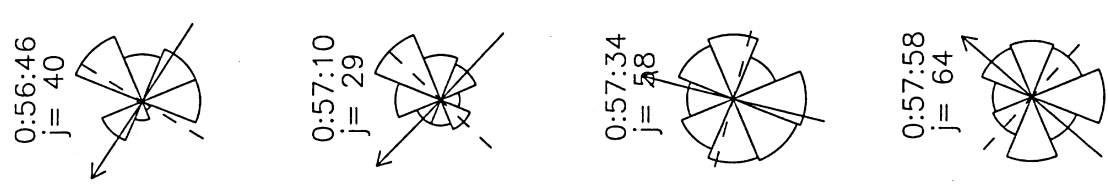

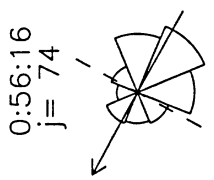
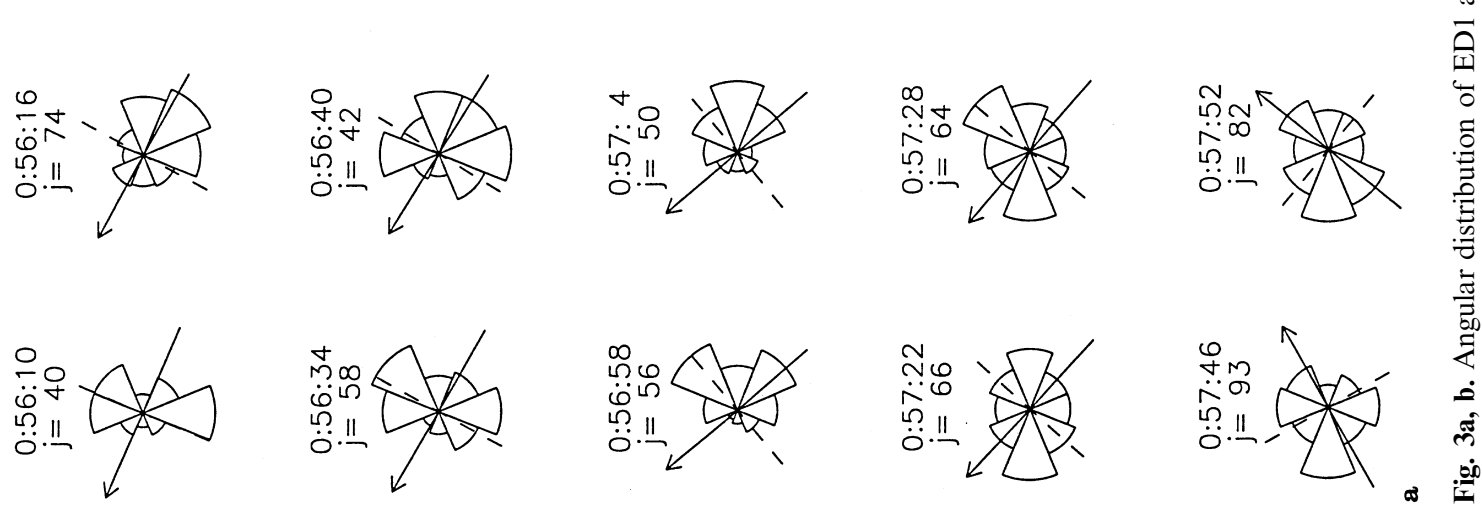


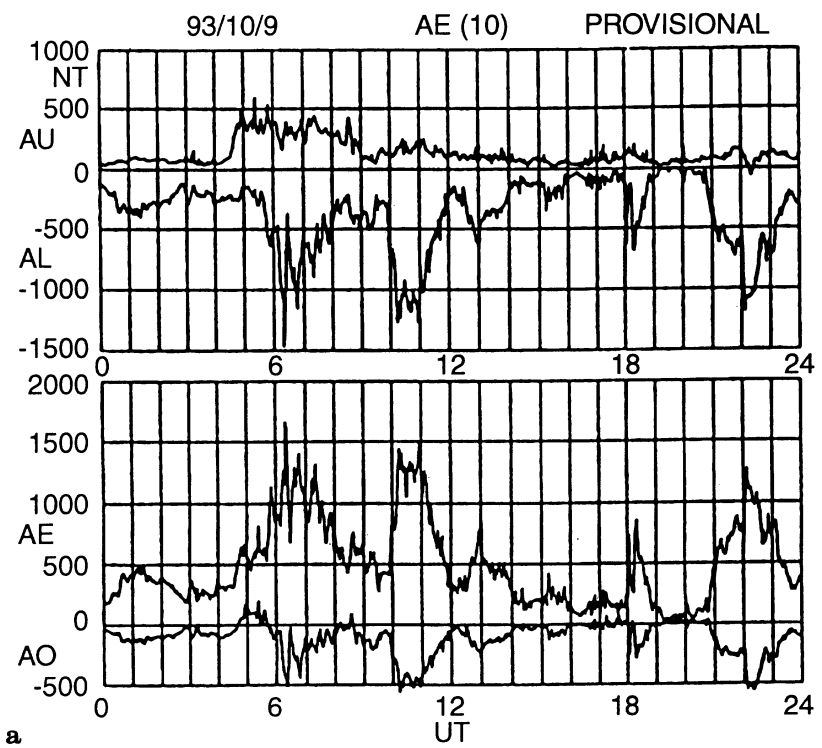

a

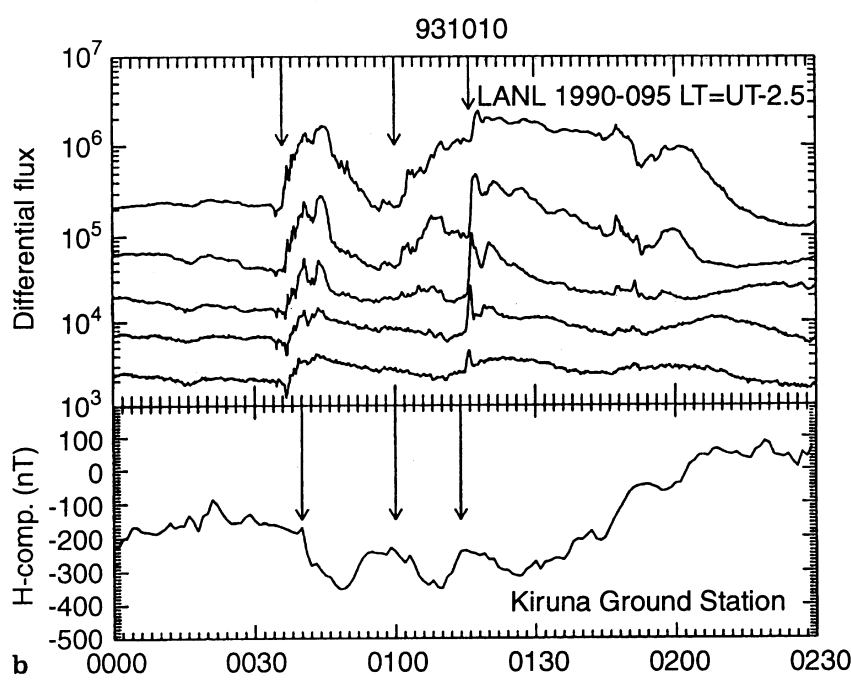

described in Fig. 2. During this event the $B x$ component decreases slightly. A bipolar perturbation is seen in the $Z$-component of the magnetic field. Just at the inflection point of $B z$, the maximum field signature due to compression of the lobe magnetic field is observed at $B x$ component, with a magnitude that exceeds slightly the value of the lobe field detected between 0115 and 0118 UT. The first dashed vertical line shown in Fig. 5 at 0123:20 UT is drawn through the maximum core field which is observed simultaneously with the bipolar turning of the $B z$ component. Moreover, a clear anisotropy of tailward versus earthward streaming ions and electrons during this BLP, can be seen in the first four panels of Fig. 5 from $\sim 0118$ UT to $\sim 0125$ UT. This is probably an instance of a boundary layer plasmoid (BLP), as defined by Moldwin and Hughes (1992b).

The magnetic field signatures presented are indicative of the PSBL (see Introduction). Nevertheless, to verify the existence of the primarily characteristic of the PSBL which is the field aligned ion beams, we examine the angular distributions of electrons and ions of different

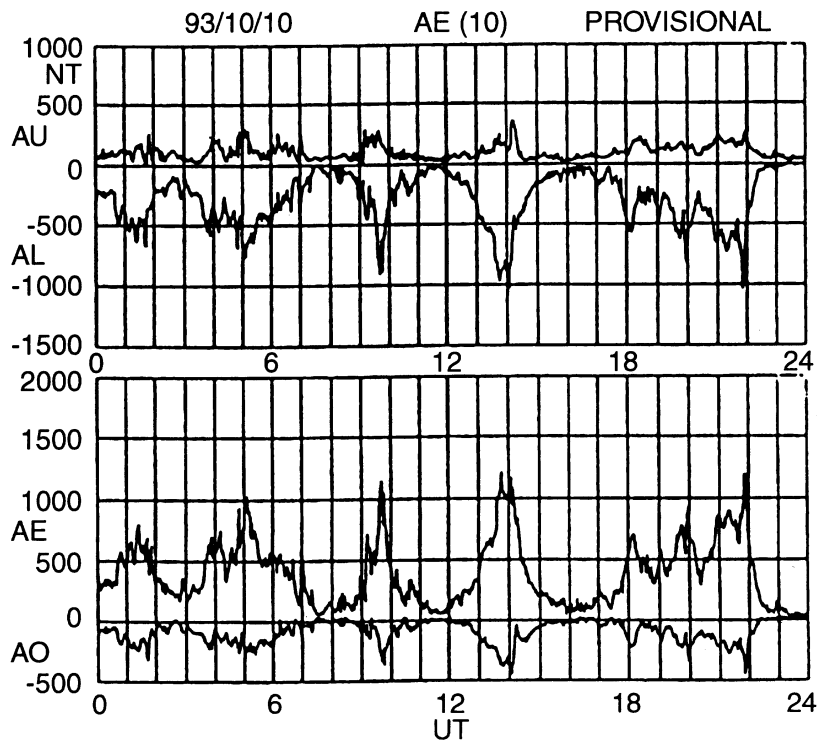

Fig. 4. a The AE indices for October 9 and 10, 1993; b differential electron fluxes as measured at geostationary orbit by LANL 1990095 spacecraft, from five channels with energy ranges $50-75 \mathrm{keV}$, 75-105 keV, 105-150 keV, 150-225 keV, 225-315 keV, plotted respectively from the top to the bottom of the upper panel, for October 10, 1993 between 0000 UT and 0230 UT. The ground magnetic field measurements from Kiruna Observatory are also presented in the lower panel, for the same interval

energies separately. The results for energetic electrons are presented in Fig. 6a using the same format as Fig. 3. A beam of electrons flowing in the tailward direction, with a very strong duskward component, appears at GEOTAIL position at 0118:40 UT. A dawn-to-dusk component appears systematically in the angular distribution plots during the entire electron beam interval, although not so intense as during the first $15 \mathrm{~s}$. The beam-like tailward electron flow lasts until 0119:43 UT. From $\sim 0120$ UT through $\sim 0124$ UT, although the count level is low, close to background values, the dominant sector of maximum counts is that looking towards the Sun. The angular distributions of energetic ions from channels E3, E4 and E5 are given in Fig. 6b, c and $\mathrm{d}$ respectively. The first beam of tailward streaming E3 ions is detected at 0119:04 UT. This high intensity beam is seen until 0124:46 UT, when the counts return back to background level. The higher energy ions detected by the E4 and E5 channels start to flow tailward at 0118:58 UT and 0118:52 UT respectively (Fig. 6c, d), and they keep flowing in such a way for $\sim 7 \mathrm{~min}$. Although the resolution of channel E5 is $48 \mathrm{~s}$, 


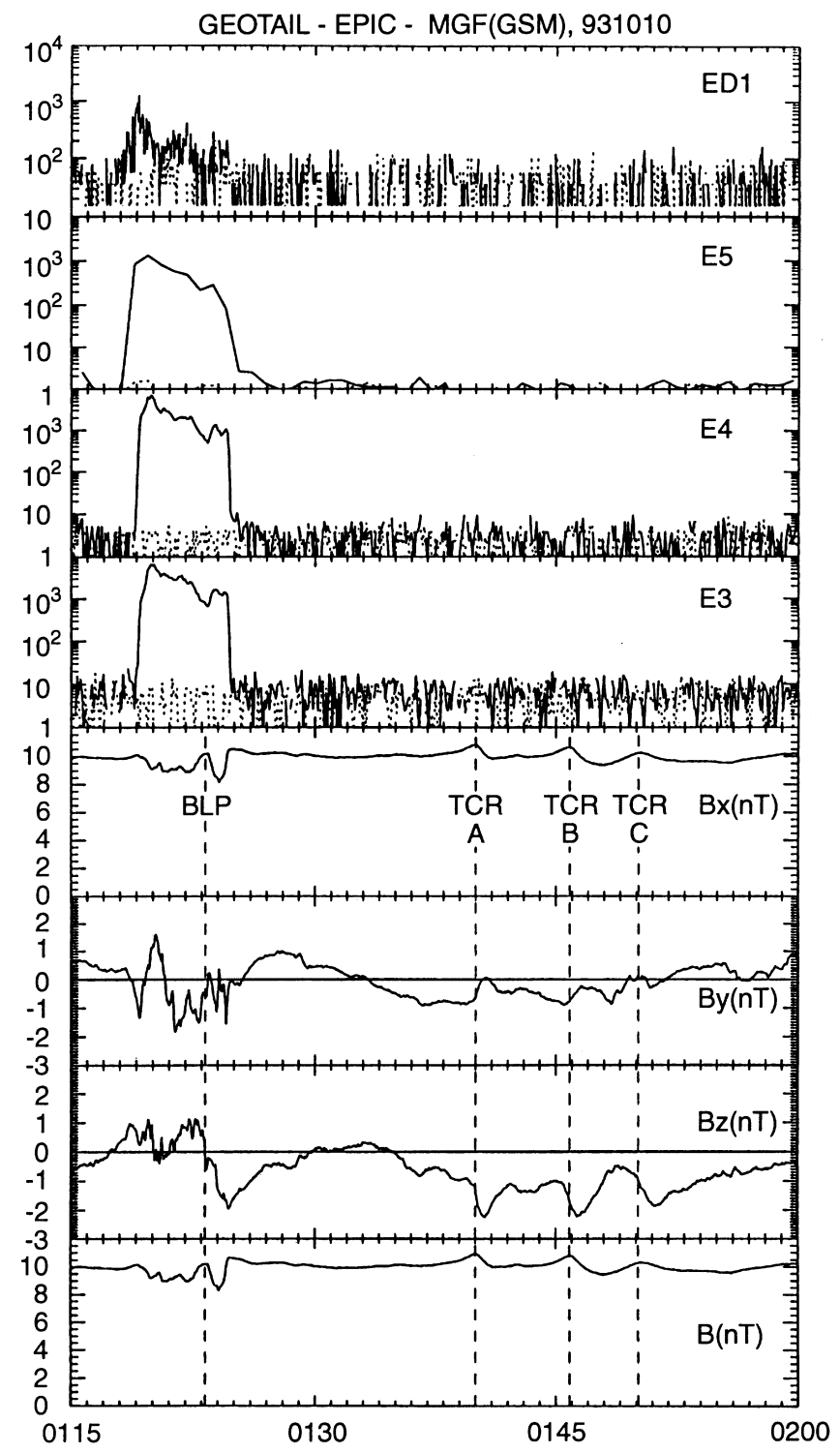

Fig. 5. An expanded overview of the time interval 0115 UT 0200 UT, where a boundary layer plasmoid (BLP) is observed following by three travelling compression regions (TCR). The dashed vertical lines are drawn through the centre of the BLP and of the TCRs

the time sequence of tailward intensification of the flow, observed by the three channels of ions of different energies, gives us strong evidence of positive velocity dispersion in the leading edge of the boundary layer plasmoid. An interesting feature that arises from the angular distribution analysis is that in the time interval between the two plasmoids, i.e., from 0115 UT to $0118 \mathrm{UT}$, the energetic ions are streaming tailward along the field lines even though the count rate is very low. This feature is indicative of the PSBL.

A second intensification of the westward electrojet at $\sim 0100$ UT during the expansion phase of the substorm detected in Kiruna is probably related to the BLP observed by GEOTAIL at $\sim 0120$ UT (see Fig. 4b).

After 0130 UT GEOTAIL detects a negative $B z$ component for $\sim 25 \mathrm{~min}$. During this interval of south- ward $B z$, three TCRs are observed at $\sim 0140 \mathrm{UT}$, $\sim 0146$ UT and $\sim 0150$ UT as is indicated in Fig. 5 by the dotted vertical lines. The $B z$ component is still to the south for $10 \mathrm{~min}$ after the last compression region. This multiple TCR event is preceded by a decrease in the $X$-component at $0116 \mathrm{UT}$ detected in the ground magnetogram of Kiruna Observatory (Fig. 4b). Although there are multiple decreases in the $X$-component following the large decrease at $0116 \mathrm{UT}$, there is no clear association between the subsequent TCRs in this multiple event and the fine structure in the $X$-component. The three compression regions are accompanied by $+B z$ then $-B z$ variations, with the peak compression being near the inflection point in the north-south component. The most intense and long duration event $(\sim 4 \mathrm{~min})$ is the first TCR, with a compression ratio of $10 \%$.

To identify better the nature of the BLP and TCRs observed by GEOTAIL we examine the magnetic field using principal axis coordinates (Sonnerup and Cahill, 1967). Figure 7 presents the principal axis analysis (PAA) of the 3-s resolution boundary layer plasmoid magnetic field measurements. In this system, B1 is the magnetic field along the direction of maximum variance, B2 is the magnetic field component along the direction of intermediate variance, and B3 is the magnetic field component along the minimum variance direction, along which the magnetic field exhibits the least change. The direction of the principal axis (i.e., the eigenvector) in GSM coordinates as well as the eigenvalues are given in each of the panels. As noted in Fig. 7, the eigenvector of the minimum variance direction B3 is $(0.246,0.787$, $0.565)$ in GSM coordinates, which lies in the $Y-Z$ plane, being closer to $Y$ axis. The magnetic field along the intermediate variance direction B2 is nearly aligned with the $X$-axis, seen from the eigenvector coordinates $(0.898$, $-0.405,0.173)$. The $\mathrm{B} 2$ component exhibits an increase in magnitude around $0123 \mathrm{UT}$, which corresponds to the peak of the magnetic field at the centre of the BLP and is oriented antiparallel to the tail axis. The axis of the maximum variance direction, B1, is essentially in the $Z$-direction $(0.365,0.465,-0.806)$ and represents the north - then - south $B z$ variation. Hodograms at the bottom of Fig. 7 provide views of the magnetic field variation in the two orthogonal planes. Here we follow the format proposed by Slavin et al. (1993) by marking the beginning and the end of the traces with "B" and "E" respectively. The hodograms show a well-defined rotation in the B2-B1 plane, which is close to $180^{\circ}$ rotation and a quite variable B3-B1 trace. Since B1 component is nearly aligned with the $Z$ axis and B2 component is nearly aligned with the $X$ axis, we come to the conclusion that the tip of the magnetic field vector during the BLP mainly rotated in the plane containing the ambient magnetic field.

As pointed out earlier, the BLP is followed by three short duration TCRs. Figure 8 displays the magnetic field components in principal axis coordinates and the PAA hodograms for the first TCR. The results from the other two TCRs are almost the same, thus they are not presented. The field presents a well-defined rotation in the B2-B1 plane with relatively stable B3 component. 

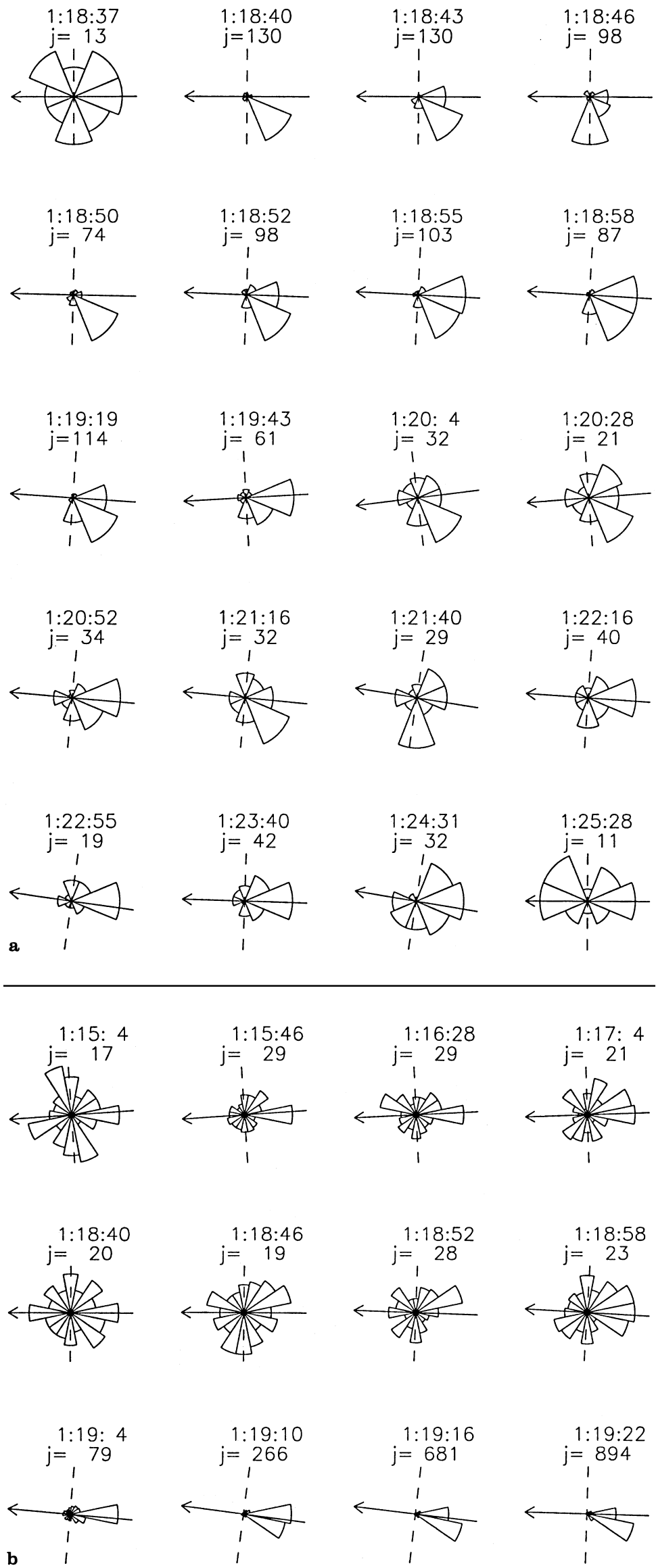

Fig. 6. a-d Angular distributions of energetic electrons with $\mathrm{E}>38 \mathrm{keV}$ in the ecliptic plane, during the BLP observed between 0115 UT and 0125 UT. The equatorial magnetic field projection is overplotted. The Sun lies to the left of the figure. b The angular distributions of $62-74 \mathrm{keV}$ ions $\left(\right.$ mostly $\mathrm{H}^{+}$), detected from channel E3, $\mathbf{c}$ the angular distributions of 74-89 $\mathrm{keV}$ ions (mostly $\mathrm{H}^{+}$), detected from channel E4, and d the angular distributions of $89-110 \mathrm{keV}$ ions (mostly $\left.\mathrm{H}^{+}\right)$, detected from channel E5 

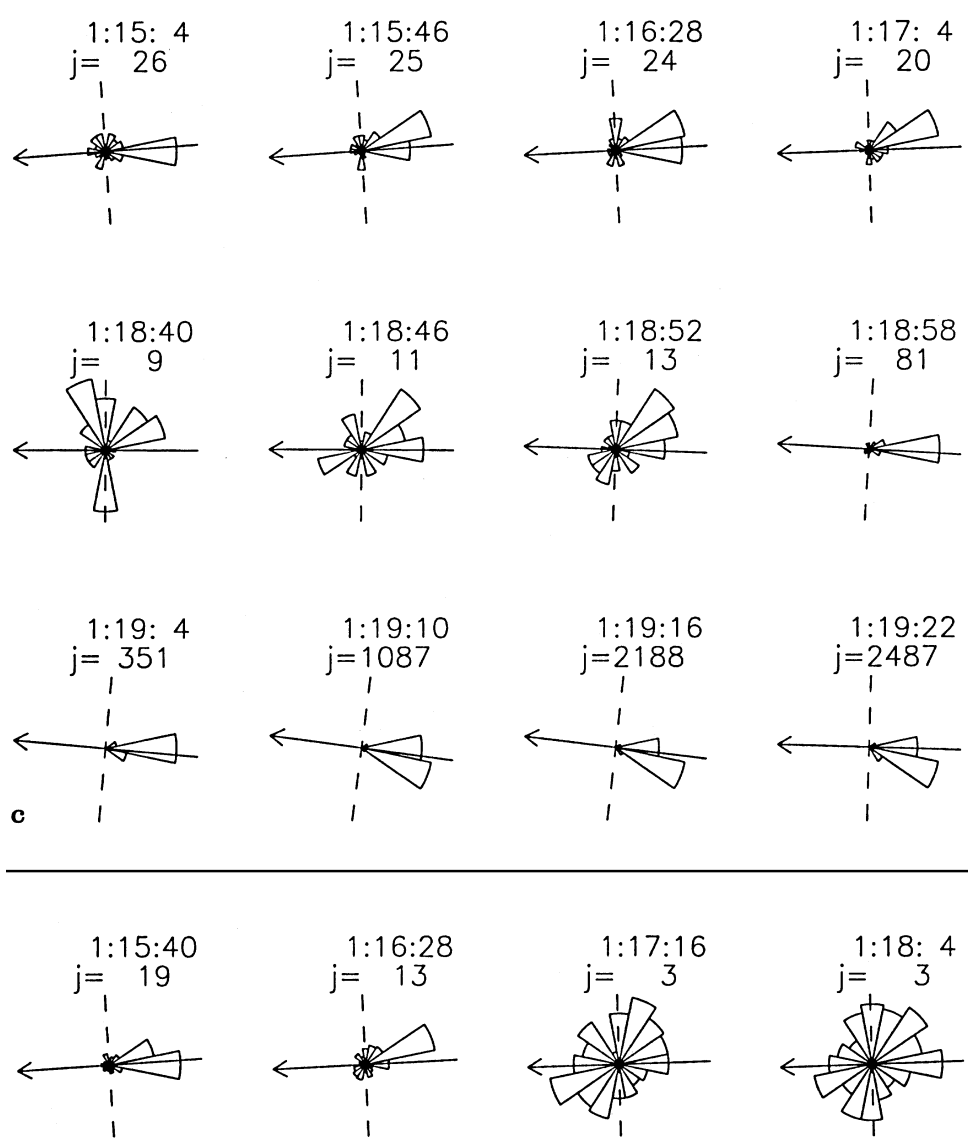

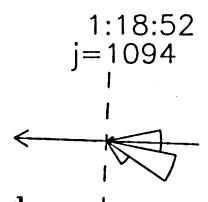

d
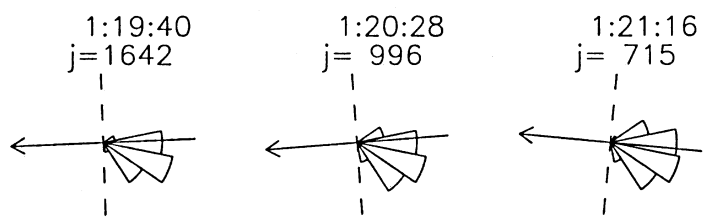

The tip of the magnetic field vector in all cases of compression regions studied, rotated in a plane containing the ambient field. The hodograms presented here are smoother than those of Fig. 7 which correspond to the BLP. This is due to the absence of high beta plasma sheet and PSBL flux tubes surrounding the plasmoids (Slavin et al., 1989). The minimum variance direction is essentially aligned with the $Y$ axis in all cases and it is consistent with the east-west centre of their associated plasmoid passing very nearly under the spacecraft. Finally, the large ratios of maximum to intermediate $(\mathrm{L} 1 / \mathrm{L} 2=9.66,4.64$ and 7.18 for TCR A, B and C respectively) and intermediate to minimum eigenvalues $(\mathrm{L} 2 / \mathrm{L} 3=47,32$ and 2.43 for $\mathrm{TCR} \mathrm{A}, \mathrm{B}$ and $\mathrm{C}$ respectively) signify that the structures are well defined.

\section{Summary of observations}

a. A large plasmoid was observed by GEOTAIL at 0055 UT. At the front edge of the structure, energetic electrons flow tailward whereas energetic ions flow earthward until tailward convected population is observed. No energy dispersion was observed.

b. The first large plasmoid leaves GEOTAIL in the boundary layer plasma sheet. This was identified by (1) the magnitude of the constant magnetic field which exceeds the lobe value by $25 \%$ and (2) the tailward field aligned streaming ions observed even at very low count rates.

c. While GEOTAIL is in the PSBL, a BLP was observed, followed by three TCRs.

d. Particle signatures observed during the boundary layer plasmoid show tailward streaming electrons during the passage of the structure. Positive velocity dispersion was observed in the front edge of the structure while reverse velocity dispersion was seen in the rear edge.

e. The principal axis analysis performed in the three TCRs revealed a very simple nature of magnetic field.

f. The first large plasma sheet plasmoid centered at $\sim 0106$ UT was associated with the substorm onset seen in ground magnetograms at 0040 UT and in particle injections at geosynchronous altitudes at $\sim 0036$ UT as well. 

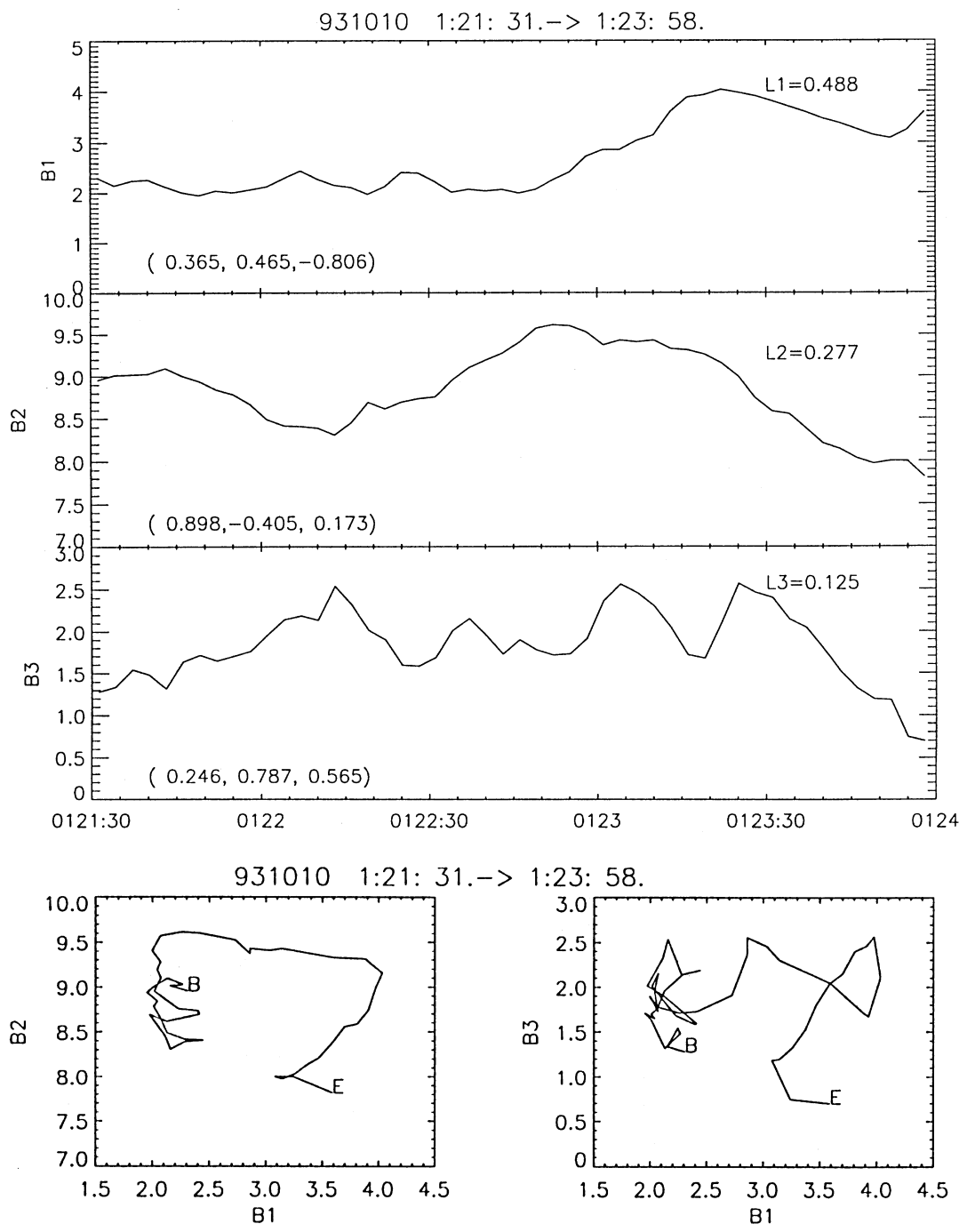

Fig. 7. $3 \mathrm{~s}$ averages of the GEOTAIL magnetic field data across the boundary layer plasmoid displayed in principal axis coordinates. Hodograms of the field variation in two orthogonal planes are shown in the bottom panels

g. The boundary layer plasmoid centered at $\sim 0123$ UT is associated with the substorm intensification seen at 0100 UT on the ground and at geosynchronous altitudes as well.

h. Finally, there was a particle injection event observed at geosynchronous altitude at 0116 UT preceding the three travelling compression regions detected by GEOTAIL at 0140 UT, 0146 UT and 0150 UT. The three TCRs were observed during the recovery phase of the substorm. There was no clear one-to-one correlation between the observed TCRs and substorm intensifications.

\section{Discussion}

We have studied the structure and dynamics of the distant magnetotail during a strong substorm. Magnetic field and energetic particle measurements from GEOTAIL spacecraft, related to this event have been examined.

The Earth's magnetotail responded to this substorm with the ejection of five plasmoids. The first of them, with the maximum duration $(\sim 20 \mathrm{~min})$ was ejected $17 \mathrm{~min}$ after the expansion onset of the substorm. GEOTAIL was engulfed by this passing plasmoid. The second plasmoid ejected $\sim 20$ min after the intensification of the westward electrojet recorded in Kiruna ground observatory at $\sim 0100 \mathrm{UT}$, had no significantly large $Z$ extent, so that GEOTAIL observed the travelling bulge in the PSBL in the form of a boundary layer plasmoid. Subsequently three small plasmoids were ejected, following an additional intensification of the westward electrojet recorded at 0116 UT. Nevertheless, no other distinct intensifications were observed after 0116 UT. The effects of these passing plasmoids were observed in the lobe by GEOTAIL in the form of travelling compression regions.

These observations are in accordance with Moldwin and Hughes (1993) results, suggesting that there was no one-to-one correspondence of the numbers of plasmoids compared to individual identifiable onsets during a period of prolonged substorm activity. In contrast Slavin et al. (1992) found one-to-one correlation between each substorm intensification and a plasmoid or a TCR observed in the deep tail by ISEE3. 

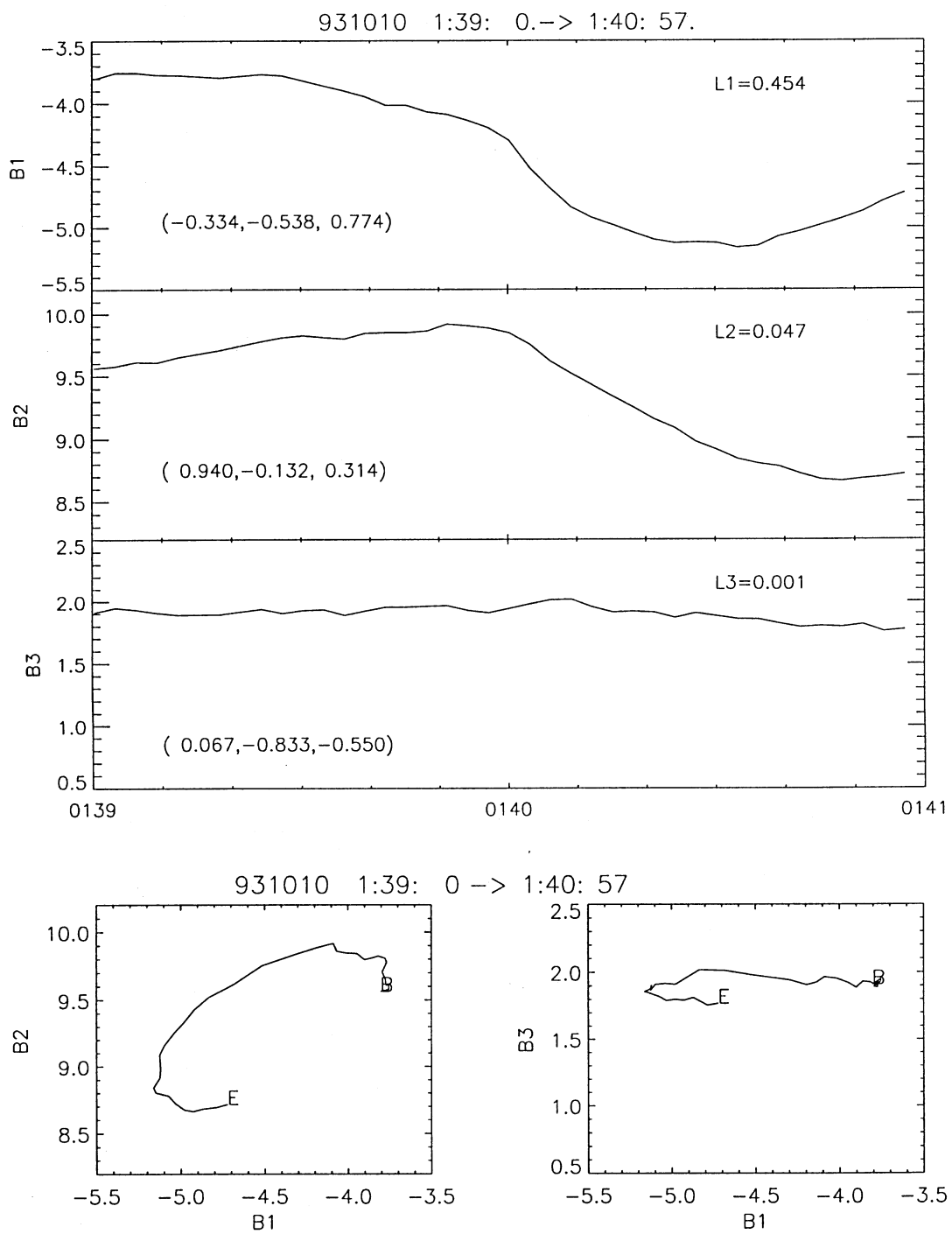

Fig. 8. $3 \mathrm{~s}$ averages of the GEOTAIL magnetic field data across the travelling compression region event $\mathrm{A}$, displayed in principal axis coordinates. Hodograms of the field variation in two orthogonal planes are shown in the bottom panels

\section{Plasmoid velocity and dynamics}

The timing of the distant tail plasmoid observation relative to the earlier substorm onset, the dimensions of the plasmoids and the bulk speed of the plasma within the magnetic structures can lead us to some conclusions concerning the dynamics of the magnetotail. Unfortunately, during the selected time interval, data from the comprehensive plasma instrument (CPI) onboard GEOTAIL, had a lot of gaps. During the first plasmoid, i.e. from 0055 UT to 0112 UT there was only one data point from the CPI experiment, indicating that the $V x$ component of the proton bulk flow reached the value of $600 \mathrm{~km} / \mathrm{s}$. The convective (bulk) speed of the suprathermal particle population after 0102 UT, when the tailward flow became more anisotropic, can be estimated indirectly from the Compton-Getting effect (Gold et al., 1975). From the measurements of the spectral index $(\gamma=3.9)$ and the anisotropy ratio (the forward versus the backward count rate $J_{f} / J_{b}=20$ ) at the start of the convection interval, the Compton-Getting transformation gives a bulk speed $\mathrm{u}=620 \mathrm{~km} / \mathrm{s}$. This is in very good agreement with the plasma data available from GEOTAIL/CPI experiment. Given that the high energy tail of the thermal ion population convected with the magnetic structure is observed for about $12 \mathrm{~min}$, the length of the magnetic structure based upon the convection speed estimated indirectly from the Compton-Getting effect is $\sim(56 \pm 11)$ Re. The uncertainty in the estimation of the plasmoid length is due to the uncertainty of the start time and end time of the convection and due to error in the estimation of the bulk speed from the Compton-Getting effect.

For the boundary layer plasmoid the downtail speed and size can be estimated from the measured duration and the time between observation at GEOTAIL and substorm onset, as was suggested originally by Moldwin and Hughes (1992b). If we assume that the near-Earth reconnection site is at $X_{N E N L}=-20 \mathrm{Re}$ and that plasmoid is released at substorm onset and propagates with a constant velocity after formation, the downtail velocity can be estimated by $V=\left(X_{\text {GEOTAIL }}-X_{N E N L}\right) /$ $\left(t_{\text {GEOTAIL }}-t_{\text {onset }}\right)$, where $X_{\text {GEOTAIL }}=-148.5 \mathrm{Re}$ is the location of GEOTAIL at time of observation, $X_{N E N L}$ is 
the location of the near-Earth reconnection site, $t_{\text {GEOTAIL }}=0127 \mathrm{UT}$ is the time of the end of the event at GEOTAIL, and $t_{\text {onset }}=0100 \mathrm{UT}$ is the time of substorm intensification (Moldwin and Hughes, 1992b). The downtail velocity for this BLP was found to be $510 \mathrm{~km} / \mathrm{s}$. The estimated size of the plasmoid can be calculated using the velocity and duration of the event by $L=V x T_{B L P}$, where $T_{B L P}$ is the duration of the BLP. The size for this event is $(28 \pm 5) \mathrm{Re}$, given that the duration of the event was estimated to be $6 \mathrm{~min}$ which represents the duration of the entire bipolar turning of $B z$-component. The uncertainty of the BLP length is (1) due to the error in the onset time which is $\pm 1 \mathrm{~min}$, (2) due to the error in the assumption of the near-Earth $X$-line position, which might continuously retreat after each successive onset and (3) due to the uncertainty in the estimation of the end time of the event at GEOTAIL position due to an amount of draping and pile-up observed before and after the magnetic structure.

To estimate the velocity and length of the compression regions which appeared at GEOTAIL during the recovery after the substorm, we follow the same method suggested by Moldwin and Hughes (1992b) which has been applied previously to estimate the bulk velocity of the BLP, given that TCRs are caused by the draping of lobe flux tubes about plasmoids moving rapidly tailward (Murphy et al., 1987; Owen and Slavin, 1992; Slavin et al., 1993). This local compression of the lobe magnetic field then accompanies the plasmoid as it moves down the tail and can be observed by GEOTAIL residing the lobes. Here, it is assumed again that the compression originates near $X_{N E N L}=-20 \mathrm{Re}$. Given that GEOTAIL was located at $X_{G S M}=-149 \mathrm{Re}$, the end of the event was observed by GEOTAIL at $t_{\text {GEOTAIL }}=$ 0141 UT and the intensification of the westward electrojet that caused the first TCR observed at $t_{\text {onset }}=$ $0116 \mathrm{UT}$, the formula proposed by Moldwin and Hughes (1992b) gives a downtail velocity of $555 \mathrm{~km} / \mathrm{s}$. The estimated size for this event is $(21 \pm 4) \mathrm{Re}$, given that the duration of the event was calculated to be 4 min, which represents the duration of the entire bipolar turning of $B z$-component. The uncertainties of the length of the plasmoid caused the first TCR to have been calculated as in the previous case, for the BLP length.

The estimated lengths of the plasmoid structures ejected downtail during the expansion phase of this substorm lead us to the conclusion that the magnetotail is responding with a series of plasmoids, whose size decreases from one plasmoid to the next, as shown in Table 2, the summary is given of computed velocities and lengths of the three types of magnetic structures (the plasma sheet plasmoid, the BLP and the first TCR) detected in the magnetotail by GEOTAIL during this isolated strong substorm. The lengths derived from the BLP and the TCR for the underlying plasmoid bulges are considerably smaller than the estimated length of the first plasmoid. This is in accordance with the results from previous studies (Moldwin and Hughes, 1992b; Slavin et al., 1993). According to Slavin et al. (1993) a TCR can only be used to sense remotely the plasmoid bulge and not the entire region magnetically connected to the plasmoid, because the distance between the extrema in the $B z$ signature used to estimate the duration, is estimated to be only about $75 \%$ of the plasmoid. The total plasmoid dimensions along the $X$ axis is therefore $\sim 36 \mathrm{Re}$ and $\sim 28 \mathrm{Re}$ for the BLP and the TCR respectively, which is again in accordance with the conclusion that the size of the individual plasmoids ejected downtail during a strong substorm, decreases from one plasmoid to the next.

\section{Magnetic topology of plasmoids}

From the study of the energetic particle angular distribution it is inferred that at the start of the event ( $~ 0055$ UT), GEOTAIL was located earthward of the distant neutral line, but close enough to detect earthward-flowing energetic particles accelerated from the distant $X$-line. Consequently, a reversal to tailward flow is observed. During the plasmoid observed from $0055 \mathrm{UT}$ to $0112 \mathrm{UT}$, no velocity dispersion was observed. This gives us additional supporting evidence for the scenario that energetic particles are accelerated from the distant $X$-line. Observations of earthward streaming ions inside a flux rope has been reported by Kawano et al. (1994) during an event observed by GEOTAIL in the middle tail and was explained by the hypothesis that energetic ions are coming from the distant $X$-line. As an alternative explanation, DeCoster and Frank (1979) referred to the possibility of a field aligned electric field in the magnetotail which accelerates ions earthward and electrons tailward.

At the centre of the structure a very large $B y$ component is observed, while energetic particles are convected tailward. According to Hughes and Sibeck (1987) the large dawn-to-dusk component results in the

Table 2. Length and TOF speed determination of TCRs

\begin{tabular}{|c|c|c|c|c|}
\hline $\begin{array}{l}\text { Type of magnetic } \\
\text { structure }\end{array}$ & Centre of the structure & TOF speed $(\mathrm{km} / \mathrm{s})$ & $\begin{array}{l}\text { Length }(\mathrm{Re}) \text { (based } \\
\text { on the duration } \\
\text { of the entire bipolar } \\
\text { turning) }\end{array}$ & $\begin{array}{l}\text { Length }(\mathrm{Re}) \text { (based on the } \\
\text { duration of the entire } \\
\text { region connected } \\
\text { magnetically } \\
\text { to the plasmoid) }\end{array}$ \\
\hline Plasmoid & 0105:50 UT & 620 & $56 \pm 11$ & $56 \pm 11$ \\
\hline Boundary layer plasmoid & 0123:20 UT & 510 & $28 \pm 5$ & $36 \pm 6$ \\
\hline Travelling compression region & $0140 \mathrm{UT}$ & 555 & $21 \pm 4$ & $28 \pm 5$ \\
\hline
\end{tabular}


formation of a flux rope magnetic structure. Their threedimensional model predicts energetic particle signatures similar to those of the two-dimensional model. When a plasmoid passes a spacecraft in the distant magnetotail, the plasma sheet expands to engulf the spacecraft, which in the Hughes and Sibeck (1987) model passes successively from open lobe field lines with one end attached to the Earth, to newly merged open boundary layer field lines with both ends in the solar wind, to interior plasmoid magnetic field lines still connected to the Earth.

The magnetic field lines of the boundary layer plasmoid ejected $5 \mathrm{~min}$ after the first large plasmoid passage, appears to be open as expected by the boundary layer plasmoid definition. This has been supported from the analysis of the angular distribution of electrons with $\mathrm{E}>38 \mathrm{keV}$, which show tailward flow during the whole interval of the event. Moreover, positive velocity dispersion was observed in the front edge of the BLP, while reverse velocity dispersion was observed at the rear edge. Thus we can adopt the Murphy et al. (1987) conclusion according to which bulk movement of the plasma sheet relative to the spacecraft, caused for example, by the tailward motion of a plasmoid, moves the energetic ion boundary layer past the spacecraft.

The principal axis analysis performed on the magnetic field during the BLP interval, shows a well-defined rotation in the B1-B2 plane, which is less than $180^{\circ}$. On the other hand the magnetic field in the B1-B3 plane, presents a more complex behaviour, showing that the magnetic field along the B3 component (in the direction of minimum variance, largely along the $Y$-axis) presents relatively large variations. The expected magnetic field variations observed by a spacecraft passing through the centre of an idealised magnetic island and a flux rope, have been examined by Slavin et al. (1989) and presented in their Fig. 11a. In a more realistic approach, a spacecraft passing well above the axis of the loop, which is the case when the spacecraft encounters a BLP, would result in a rotation of less than $180^{\circ}$ while motion of the plasmoid relative to the spacecraft can produce much larger rotations.

The results obtained from the PAA performed on the magnetic field data during the three TCR intervals show a well-defined rotation of the field in B1-B2 plane and an almost linear variation of the field in the B1-B3 plane, along the axis of the maximum variation which is almost aligned with the $Z$ axis. This $2 \mathrm{D}$ perturbation in a plane almost coincident with the $X-Z$ plane, verifies that the spacecraft observes the tilting of the lobe field due to the passage of a plasmoid underneath. Thus, the peak in the intermediate field direction (along the $X$-axis) is due to the compression of the lobe field.

\section{Conclusions}

We presented a case study of the magnetotail response during an intense substorm, which is part of a longer period of disturbance. The analysis of energetic particle and magnetic field data from the deep tail phase of the
GEOTAIL mission together with data from geosynchronous altitudes and ground magnetometers, showed that a series of plasmoids are generated, with each roughly corresponding to a substorm intensification and each being smaller than the previous one. This latter conclusion is supported in that GEOTAIL first observes a flux rope plasmoid in the plasma sheet, then a boundary layer plasmoid and then finally a series of three travelling compression regions in the lobes. These comprehensive new observations, taken during a period of prolong substorm activity, give full support to the model of multiple plasmoid injection (Slavin et al., 1993; Moldwin and Hughes, 1993) and contradict the classic plasmoid model (Hones, 1976).

Acknowledgements. Thanks are due to Stuart Nylund for the EPIC/ GEOTAIL data processing. We are grateful to Geoff Reeves of the LANL energetic particle team, for providing us with the differential electron fluxes by LANL 1990-095 spacecraft.

Topical Editor K.-H. Glaßmeier thanks M.B. Moldwin and another referee for their help in evaluating this paper.

\section{References}

Baker, D. N., S. J. Bame, R. D. Belian, W. C. Feldman, J. T. Gosling, P. R. Higbie, E. W. Hones, Jr., D. J. McComas, and R. D. Zwickl, Correlated dynamical changes in the near-Earth and distant magnetotail regions: ISEE3, J. Geophys. Res., 89, 38553864, 1984.

Baker, D. N., R. C. Anderson, R. D. Zwickl, and J. A. Slavin, Average plasma and magnetic field variations in the distant magnetotail associated with near-Earth substorm effects, J. Geophys. Res., 92, 71-81, 1987.

Belehaki, A., E. T. Sarris, G. Tsiropoula, R. W. McEntire, S. Kokubun, and T. Yamamoto, Energetic particle bursts detected by GEOTAIL in the distant tail, Proc. of the ICS-3, ESA SP389, 487-492, 1996

Belehaki, A., E. T. Sarris, G. Tsiropoula, R. W. McEntire, S. Kokubun, and T. Yamamoto, Observations of flux rope-associated particle bursts with GEOTAIL in the distant tail, Annales Geophys., in press, 1997.

DeCoster, R. J., and L. A. Frank, Observations pertaining to the dynamics of the plasma sheet, J. Geophys. Res., 84, 5099, 1979.

Frank, L. A., W. R. Paterson, K. L. Ackerson, S. Kokubun, T. Yamamoto, D. H. Fairfield, and R. P. Lepping, Observations of plasmas associated with the magnetic signature of a plasmoid in the distant magnetotail, Geophys. Res. Lett., 21, 2967, 1994.

Gold, R. E., C. O. Bostrom, and E. C. Roelof, Anisotropy measurements of $\sim 50 \mathrm{keV}$ solar protons, Conf. Pap. Int. Cosmic Ray Conf. $14^{\text {th }}, \mathbf{5}, 1801,1975$.

Hones, E. W., Jr., The magnetotail: its generation and dissipation, in Physics of solar planetary environments, Ed. D.J. Williams, 557-571, AGU, Washington D.C., 1976.

Hones, E. W., Jr., Transient phenomena in the magnetotail and their relation to substorms, Space Sci. Rev. 23, 393, 1979.

Hones E. W., Jr, D. N. Baker, S. J. Bame, W. C. Feldman, J. T. Gosling, D. J. McComas, R. D. Zwickl, J. A. Slavin, E. J. Smith, and B. T. Tsurutani, Structure of the magnetotail at $220 \mathrm{R}_{\mathrm{E}}$ and its response to geomagnetic activity, Geophys. Res. Lett., 11, 5-7, 1984a.

Hones E. W., Jr, J. Birn, D. N. Baker, S. J. Bame, W. C. Feldman, D. J. McComas, and R. D. Zwickl, Detailed examination of a plasmoid in the distant magnetotail with ISEE 3, Geophys. Res. Lett., 11, 1046, 1984b.

Hughes, W. J., and D. G. Sibeck, On the 3-dimensional structure of plasmoids, Geophys. Res. Lett., 14, 636-639, 1987. 
Kawano, H., T. Yamamoto, S. Kokubun, K. Tsuruda, A. T. Y. Lui, D. J. Williams, K. Yumoto, H. Hayakawa, M. Nakamura, T. Okada, A. Mutsuoka, K. Shiokawa, and A. Nishida, A flux rope followed by recurring encounters with traveling compression regions: GEOTAIL observations, Geophys. Res. Lett., 21, 2891, 1994.

Kokubun, S., T. Yamamoto, M. H. Acuna, K. Hayashi, K. Shiokawa, and H. Kawano, The GEOTAIL magnetic field experiment, J. Geomag. Geoelectr., 46, 7-21, 1994.

Lui, A. T. Y., D. J. Williams, S. P. Christon, R. W. McEntire, V. Angelopoulos, C. Jacquey, T. Yamamoto, and S. Kokubun, A preliminary assessment of energetic ion species in flux ropes/ plasmoids in the distant tail, Geophys. Res. Lett., 21, 3019-3022, 1994.

Machida, S., T. Mukai, Y. Saito, T. Obara, T. Yamamoto, and S. Kokubun, Geotail low energy particle and magnetic field observations of a plasmoid at $X=-142$ Re, Geophys. Res. Lett., 21, 295, 1994.

Moldwin M. B., and W. J. Hughes, A study of plasmoid properties to examine the formation and evolution of plasmoids: survey of ISEE3 Geotail data, J. Geophys. Res., 97, 19259, 1992a.

Moldwin M. B., and W. J. Hughes, Plasmoid observations in the distant plasma sheet boundary layer, Geophys. Res. Lett., 19, 1911-1914, 1992b.

Moldwin M. B., and W. J. Hughes, Geomagnetic substorm association of plasmoids, J. Geophys. Res., 98, 81-88, 1993.

Murphy N., J. A. Slavin, D. N. Baker, and W. J. Hughes, Enhancements of energetic ions associated with travelling compression regions in the deep geomagnetic tail, J. Geophys. Res., 92, 64-70, 1987.

Owen, C. J., and J. A. Slavin, Energetic ion events associated with travelling compression regions, in Proc. of the International Conference on Substorms - 1, Eur. Space Agency Spec. Publ., ESA SP-000, 365-370, 1993.

Owen, C. J., and S. W. H. Cowley, Simple models of timedependent reconnection in a collision-free plasma with an application to substorms in the geomagnetic tail, Planet. Space Sci., 35, 451, 1987.

Richardson, I. G., and S. W. H. Cowley, Plasmoid-associated energetic ion bursts in the deep geomagnetic tail: properties of the boundary layer, J. Geophys. Res., 90, 12133-12155, 1985.

Richardson, I. G., S. W. H. Cowley, E. W. Hones, Jr., and S. J. Bame, Plasmoid associated energetic ion bursts in the deep geomagnetic tail: Properties of plasmoids and the postplasmoid plasma sheet, J. Geophys. Res., 92, 9997, 1987.
Scholer, M., G. Gloeckler, B. Klecker, F. M. Ipavich, D. Hovestadt, and E. J. Smith, Fast moving plasma structures in the distant magnetotail, J. Geophys. Res., 89, 6717-6727, 1984a.

Scholer, M., G. Gloeckler, D. Hovestadt, B. Klecker, and F. M. Ipavich, Characteristics of plasmoidlike structures in the distant magnetotail, J. Geophys. Res., 89, 8872-8876, 1984b.

Scholer, M., D. Hovestadt, B. Klecker, G. Gloeckler, and F. M. Ipavich, Average flow between $70 \mathrm{Re}$ and $200 \mathrm{Re}$ in the geomagnetic tail, Geophys. Res. Lett., 11, 343, 1984c.

Slavin, J. A., E. J. Smith, B. T. Tsurutani, D. G. Sibeck, H. J. Singer, D. N. Baker, J. T. Gosling, E. W. Hones, and F. L. Scarf, Substorm associated traveling compression regions in the distant tail: ISEE-3 geotail observations, Geophys. Res. Lett., 11, 657-660, 1984.

Slavin, J. A., D. N. Baker, J. D. Craven, R. C. Elphic, D. H. Fairfield, L. A. Frank, A. B. Galvin, H. J. Hughes, R. H. Manka, D. G. Mitchell, I. G. Richardson, T. R. Sanderson, D. G. Sibeck, E. J. Smith, and R. D. Zwickl, CDAW-8 observations of plasmoid signatures in the geomagnetic tail: an assessment, J. Geophys. Res., 94, 15153-15175, 1989.

Slavin, J. A., M. F. Smith, E. L. Mazur, D. N. Baker, T. Iyemori, H. J. Singer, and E. W. Greenstadt, ISEE3 plasmoid and TCR observations during an extended interval of substorm activity, Geophys. Res. Lett., 19, 825-828, 1992.

Slavin, J. A., M. F. Smith, E. L. Mazur, D. N. Baker, E. W. Hones, Jr., T. Iyemori, and E. W. Greenstadt, ISEE3 observations of travelling compression regions in the Earth's magnetotail, J. Geophys. Res., 98, 15425-15446, 1993.

Sonnerup, B. U. O., and L. J. Cahill, Magnetopause structure and attitude from Explorer 12 observations, J. Geophys. Res., 72, 171, 1967.

Tsurutani, B. T., I. G. Richardson, R. M. Thorne, W. Butler, E. J. Smith, S. W. H. Cowley, S. P. Gary, S. -I. Akasofu, and R. D. Zwickl, Observations of the right-hand resonant ion beam instability in the distant plasma sheet boundary layer, J. Geophys. Res., 90, 12159, 1985.

Williams, D. J., R. W. McEntire, C. Schlemm II, A. T. Y. Lui, G. Gloeckler, S. P. Christon, and F. Gliem, GEOTAIL energetic particles and ion composition instrument, J. Geomag. Geoelectr., 46, 39-57, 1994.

Zwickl, R. D., D. N. Baker, S. J. Bame, W. C. Feldman, J. T. Gosling, E. W. Hones, Jr., and D. J. McComas, Evolution of the Earth's distant magnetotail: ISEE3 electron plasma results, J. Geophys. Res., 89, 11007-11012, 1984. 